\title{
Charm, bottom, and quarkonia cross sections for double and triple-parton scatterings in high-energy proton-nucleus and nucleus-nucleus collisions
}

\author{
David d'Enterria \\ CERN, EP Department, 1211 Geneva, Switzerland \\ E-mail: david.d'enterrialcern.ch
}

\author{
Alexander M. Snigirev* \\ Skobeltsyn Institute of Nuclear Physics, Lomonosov Moscow State University, 119991, Moscow, \\ Russia, and \\ Bogoliubov Laboratory of Theoretical Physics, JINR, 141980, Dubna, Russia \\ E-mail: Alexandre.Sniguirev@cern.ch
}

\begin{abstract}
The framework to compute the cross sections for the production of particles with high mass and/or large transverse momentum in double- (DPS), triple- (TPS), and in general n-parton scatterings from the corresponding single-parton $\left(\sigma_{\mathrm{SPS}}\right)$ values in high-energy proton and nuclear collisions is reviewed. The basic parameter of the factorized n-parton scattering ansatz is an effective cross section $\sigma_{\text {eff }}$ encoding all unknowns about the underlying generalized n-parton distribution in the proton (nucleon). In its simplest and most economical form, the $\sigma_{\text {eff }}$ parameter can be derived from the transverse parton profile of the colliding protons and/or nucleus, using a Glauber approach. Numerical examples for the cross sections and yields expected for the concurrent DPS or TPS production of heavy-quarks, quarkonia, and/or gauge bosons in proton and nuclear collisions at LHC and Future Circular Collider (FCC) energies are provided. The obtained cross sections are based on perturbative QCD predictions for $\sigma_{\mathrm{SPS}}$ at next-to-leading-order (NLO) or next-to-NLO (NNLO) accuracy including, when needed, nuclear modifications of the corresponding parton densities.
\end{abstract}

International Conference on Hard and Electromagnetic Probes of High-Energy Nuclear Collisions 30 September - 5 October 2018

Aix-Les-Bains, Savoie, France

*Speaker. 


\section{Introduction}

Multiparton interactions are a major contributor to particle production in proton and nuclear collisions at high center-of-mass energies. The possibility to concurrently produce multiple particles with large transverse momentum and/or mass in independent parton-parton scatterings in a given proton (nucleon) collision increases with $\sqrt{s}$, and provides valuable information on the badlyknown 3D partonic profile of hadrons, on the unknown energy evolution of the parton density as a function of impact parameter $b$, and on the role of partonic spatial, momentum, flavour, colour,... correlations in the hadronic wave functions. This talk is mainly based on our studies $[1,2,3]$, which we summarize here.

In a generic hadronic collision, the inclusive cross section, $\sigma_{h h^{\prime} \rightarrow a_{1} \ldots a_{n}}^{\mathrm{NPS}}$, to produce $n$ hard particles in $n$ independent hard parton scatterings, $h h^{\prime} \rightarrow a_{1} \ldots a_{n}$, can be written, without any loss of generality, as the $n$ th-product of the corresponding single-parton scattering (SPS) cross sections, $\sigma_{h h^{\prime} \rightarrow a_{i}}^{\mathrm{SPS}}$, for the production of each single final-state particle, normalized by the $(n-1)$ th power of an effective cross section [1],

$$
\sigma_{h h^{\prime} \rightarrow a_{1} \ldots a_{n}}^{\mathrm{NPS}}=\left(\frac{m}{n !}\right) \frac{\sigma_{h h^{\prime} \rightarrow a_{1}}^{\mathrm{SPS}} \cdots \sigma_{h h^{\prime} \rightarrow a_{n}}^{\mathrm{SPS}}}{\sigma_{\mathrm{eff}, \mathrm{NPS}}^{n-1}} .
$$

The combinatorial $(m / n !)$ prefactor takes into account the different cases of (indistinguishable or not) final states. For a set of identical particles (i.e., when $a_{1}=\ldots=a_{n}$ ) we have $m=1$, whereas $m=2,3,6, \ldots$ for final-states with an increasing number of different particles produced. For the typical cases of phenomenological interest, we have

- Double-parton scattering (DPS): $m=1$ if $a_{1}=a_{2}$; and $m=2$ if $a_{1} \neq a_{2}$.

- Triple-parton scattering (TPS): $m=1$ if $a_{1}=a_{2}=a_{3} ; m=3$ if $a_{1}=a_{2}$, or $a_{1}=a_{3}$, or $a_{2}=a_{3}$; and $m=6$ if $a_{1} \neq a_{2} \neq a_{3}$.

The effective parameter $\sigma_{\text {eff,NPS }}$ encodes all the unknowns related to the underlying generalized parton distribution functions (PDFs). Equation (1.1) encapsulates the intuitive result that the probability to produce $n$ particles in a given inelastic hadron-hadron collision should be proportional to the $n$-product of probabilities to independently produce each one of them, normalized by the $(n-1)$ th power of an effective cross section to guarantee the proper units of the final result.

In the simplest baseline approach that assumes that the generalized PDFs can be factorized in terms of longitudinal and transverse components (i.e., neglecting space, momentum, flavour, colour correlations in the hadronic wave functions) [1], the effective NPS cross section bears a simple geometric interpretation in terms of powers of the inverse of the integral of the hadronhadron overlap function $T(\mathbf{b})$ over all impact parameters $b$,

$$
\sigma_{\mathrm{eff}, \mathrm{NPS}}=\left\{\int d^{2} b T^{n}(\mathbf{b})\right\}^{-1 /(n-1)} .
$$

In the proton-proton case, making use of the expressions (1.2), for a wide range of proton transverse overlap function $T(\mathbf{b})$, we found [2] a simple relationship between the effective DPS and TPS cross sections:

$$
\sigma_{\text {eff, TPS }}=(0.82 \pm 0.11) \cdot \sigma_{\text {eff,DPS }},
$$


which, for the typical $\sigma_{\text {eff,DPS }}=15 \pm 5 \mathrm{mb}$ values extracted from a broad range of DPS measurements at Tevatron and LHC, translates into

$$
\sigma_{\text {eff, TPS }}=12.5 \pm 4.5 \mathrm{mb} .
$$

This data-driven numerical value allows for the computation of any DPS and TPS cross sections in pp collisions via Eq. (1.1) $(n=2,3)$, once the corresponding SPS cross section is known.

\section{Proton-nucleus collisions}

The larger transverse parton density in nuclei compared to protons results in enhanced DPS cross sections, $\mathrm{pA} \rightarrow a b$, coming from interactions where the two partons of the nucleus belong to the same nucleon, and two different nucleons $[4,5]$. The final factorized DPS formula in protonnucleus collisions can be written as a function of the elementary proton-nucleon single-parton cross sections, $\sigma_{\mathrm{pN} \rightarrow a}^{\mathrm{SPS}}$, as [5]

$$
\sigma_{\mathrm{pA} \rightarrow a b}^{\mathrm{DPS}}=\left(\frac{m}{2}\right) \frac{\sigma_{\mathrm{pN} \rightarrow a}^{\mathrm{SPS}} \cdot \sigma_{\mathrm{pN} \rightarrow b}^{\mathrm{SPS}}}{\sigma_{\mathrm{eff}, \mathrm{DPS}, \mathrm{pA}}},
$$

where the effective DPS pA cross section in the denominator depends on the effective cross section measured in pp, and on a pure geometric quantity (nuclear overlap function $T_{\mathrm{AA}}(0)$ ) that is directly derivable from the well-known nuclear transverse profile, namely

$$
\sigma_{\mathrm{eff}, \mathrm{DPS}, \mathrm{pA}}=\frac{\sigma_{\mathrm{eff}, \mathrm{DPS}}}{A+\sigma_{\mathrm{eff}, \mathrm{DPS}} T_{\mathrm{AA}}(0)} \approx \frac{\sigma_{\mathrm{eff}, \mathrm{DPS}}}{A+A^{4 / 3} / \pi} .
$$

For a $\mathrm{Pb}$ nucleus (with $A=208$, and $T_{\mathrm{AA}}(0)=30.25 \mathrm{mb}^{-1}$ ) one obtains $\sigma_{\text {eff,DPS,pA }}=22.5 \pm 2.3 \mu \mathrm{b}$. The overall increase of DPS cross sections in pA compared to pp collisions is $\sigma_{\text {eff,DPS }} / \sigma_{\text {eff,DPS,pA }} \approx$ $\left[A+A^{4 / 3} / \pi\right]$ which, in the case of $\mathrm{pPb}$ implies a factor of $\sim 600$ relative to $\mathrm{pp}$, i.e., a factor of $\left[1+A^{1 / 3} / \pi\right] \approx 3$ higher than the naive expectation assuming the same $A$-scaling of the singleparton cross sections.

Similarly to the DPS case, the final formula for TPS in proton-nucleus reads [3]

$$
\sigma_{\mathrm{pA} \rightarrow a b c}^{\mathrm{TPS}}=\left(\frac{m}{6}\right) \frac{\sigma_{\mathrm{pN} \rightarrow a}^{\mathrm{SPS}} \cdot \sigma_{\mathrm{pN} \rightarrow b}^{\mathrm{SPS}} \cdot \sigma_{\mathrm{pN} \rightarrow c}^{\mathrm{SPS}}}{\sigma_{\mathrm{eff}, \mathrm{TPS}, \mathrm{pA}}^{2}}
$$

with

$$
\sigma_{\mathrm{eff}, \mathrm{TPS}, \mathrm{pA}} \approx\left[\frac{A}{\sigma_{\mathrm{eff}, \mathrm{TPS}}^{2}}+\frac{A^{4 / 3}}{5.7[\mathrm{mb}] \pi \sigma_{\mathrm{eff}, \mathrm{TPS}}}+\frac{A^{5 / 3}}{160\left[\mathrm{mb}^{2}\right] \pi^{2}}\right]^{-1 / 2}
$$

For a $\mathrm{Pb}$ nucleus $(A=208)$ the effective TPS cross section amounts to $\sigma_{\text {eff,TPS,pA }}=0.29 \pm 0.04 \mathrm{mb}$. For $\mathrm{pPb}$ the relative importance of the three TPS contributions is $1: 4.54: 3.56$. Namely, in $\mathrm{pPb}$ collisions, $10 \%$ of the TPS yields come from partonic interactions within just one nucleon of the lead nucleus, 50\% involve scatterings within two nucleons, and $40 \%$ come from partonic interactions in three different $\mathrm{Pb}$ nucleons. The sum of the three contributions in Eq. (2.4), ignoring differences between $\mathrm{pN}$ and $\mathrm{pp}$ collisions, indicates that the TPS cross sections in $\mathrm{pPb}$ are about nine times larger than the naive expectation based on $A$-scaling of the corresponding $\mathrm{pN}$ TPS cross sections. 


\section{Nucleus-nucleus collisions}

In nucleus-nucleus collisions, the parton flux is enhanced by $A$ nucleons in each nucleus, and the final DPS cross section "pocket formula" in heavy-ion collisions can be written as [6]

$$
\sigma_{\mathrm{AA} \rightarrow a b}^{\mathrm{DPS}}=\left(\frac{m}{2}\right) \frac{\sigma_{\mathrm{NN} \rightarrow a}^{\mathrm{SPS}} \cdot \sigma_{\mathrm{NN} \rightarrow b}^{\mathrm{SPS}}}{\sigma_{\mathrm{eff}, \mathrm{DPS}, \mathrm{AA}}}
$$

with the effective AA normalization cross section amounting to

$$
\sigma_{\mathrm{eff}, \mathrm{DPS}, \mathrm{AA}} \approx \frac{1}{A^{2}\left[\sigma_{\mathrm{eff}, \mathrm{DPS}}^{-1}+\frac{2}{A} \mathrm{~T}_{\mathrm{AA}}(0)+\frac{1}{2} \mathrm{~T}_{\mathrm{AA}}(0)\right]} .
$$

For nuclei with mass numbers $A=40-240$, we find that the relative weights of the three components contributing to DPS scattering in AA collisions are $1: 2.3: 23$ (for $A=40$ ) and $1: 4: 200$ (for $A=$ 208). Clearly, the "pure" DPS contributions arising from partonic collisions within a single nucleon are much smaller than the term from double particle production coming from two independent nucleon-nucleon collisions. The DPS cross sections in AA are practically unaffected by the value of $\sigma_{\text {eff,DPS }}$, but dominated instead by double-parton interactions from different nucleons in both nuclei.

The estimates presented in [1] demonstrate that both double- and triple- (hard) nucleon-nucleon scatterings represent a significant fraction of the inelastic hard AA cross sections, and the standard Glauber MC provides a simple approach to compute their occurrence in a given heavy-ion collision. One thus concludes that DPS and TPS processes in AA collisions are not so sensitive to the underlying $\sigma_{\text {eff,DPS }}$ (or $\sigma_{\text {eff,TPS }}$ ) values, that characterizes the intranucleon partonic structure, as they are in pp or pA collisions.

\section{Numerical examples}

The formalism above was applied in Refs $[2,3,5,6,7,8]$ to calculate the cross sections and yields expected for the concurrent DPS or TPS production of heavy-quarks, quarkonia, and/or gauge bosons in proton and nuclear collisions at LHC and Future Circular Collider (FCC) energies. Some numerical examples for resulting cross sections are listed in Tables 1 (quarkonia and gauge bosons) [1] and 2 (charm and bottom) [3] for proton-nucleus collisions.

Table 1: Production cross sections for the various DPS final states with quarkonia and electroweak bosons in $\mathrm{pPb}$ collisions at $\sqrt{s_{N N}}=8.8 \mathrm{TeV}$. The relevant SPS cross sections are also given. Uncertainties, not quoted, are of the order of $30 \%$.

\begin{tabular}{lcccc}
\hline $\mathrm{pPb}(8.8 \mathrm{TeV})$ & $J / \psi+J / \psi$ & $J / \psi+\Upsilon$ & $J / \psi+\mathrm{W}$ & $J / \psi+\mathrm{Z}$ \\
\hline$\sigma_{\mathrm{pN} \rightarrow a}^{\mathrm{SPS}}, \sigma_{\mathrm{pN} \rightarrow b}^{\mathrm{SPS}}$ & $45 \mu \mathrm{b}(\times 2)$ & $45 \mu \mathrm{b}, 2.6 \mu \mathrm{b}$ & $45 \mu \mathrm{b}, 60 \mathrm{nb}$ & $45 \mu \mathrm{b}, 35 \mathrm{nb}$ \\
$\sigma_{\mathrm{pPb}}^{\mathrm{DPS}}$ & $45 \mu \mathrm{b}$ & $5.2 \mu \mathrm{b}$ & $120 \mathrm{nb}$ & $70 \mathrm{nb}$ \\
\hline & $\Upsilon+\Upsilon$ & $\Upsilon+\mathrm{W}$ & $\Upsilon+\mathrm{Z}$ & $\mathrm{ss} \mathrm{WW}$ \\
\hline$\sigma_{\mathrm{pN} \rightarrow a}^{\mathrm{SPS}}, \sigma_{\mathrm{pN} \rightarrow b}^{\mathrm{SPS}}$ & $2.6 \mu \mathrm{b}(\times 2)$ & $2.6 \mu \mathrm{b}, 60 \mathrm{nb}$ & $2.6 \mu \mathrm{b}, 35 \mathrm{nb}$ & $60 \mathrm{nb}(\times 2)$ \\
$\sigma_{\mathrm{pPb}}^{\mathrm{DPS}}$ & $150 \mathrm{nb}$ & $7 \mathrm{nb}$ & $4 \mathrm{nb}$ & $150 \mathrm{pb}$ \\
\hline
\end{tabular}


Table 2: Cross sections for inclusive inelastic, and for SPS and TPS charm and bottom production in $\mathrm{pPb}$ (at LHC and FCC energies) and pAir (at GZK-cutoff c.m. energies) collisions. Uncertainties quoted for the SPS and TPS cross sections include scales, PDF, and total (quadratically added, including $\sigma_{\text {eff,TPS }}$ ) values.

\begin{tabular}{lccc}
\hline Process & $\mathrm{pPb}(8.8 \mathrm{TeV})$ & $\mathrm{pPb}(63 \mathrm{TeV})$ & $\mathrm{p}-\operatorname{Air}(430 \mathrm{TeV})$ \\
\hline$\sigma_{\mathrm{pA}}^{\text {inel }}$ & $2.2 \pm 0.4 \mathrm{~b}$ & $2.4 \pm 0.4 \mathrm{~b}$ & $0.61 \pm 0.10 \mathrm{~b}$ \\
\hline$\sigma_{c \bar{c} S}^{S P S}$ & $0.96 \pm 0.45_{\mathrm{sc}} \pm 0.10_{\mathrm{pdf}} \mathrm{b}$ & $3.4 \pm 1.9_{\mathrm{sc}} \pm 0.4_{\mathrm{pdf}} \mathrm{b}$ & $0.75 \pm 0.5_{\mathrm{sc}} \pm 0.1_{\mathrm{pdf}} \mathrm{b}$ \\
$\sigma_{c \bar{c}, c \bar{c}, c \bar{c}+X}^{T P S}$ & $200 \pm 140_{\text {tot }} \mathrm{mb}$ & $8.7 \pm 6.2_{\text {tot }} \mathrm{b}$ & $5.0 \pm 3.6_{\text {tot }} \mathrm{b}$ \\
\hline$\sigma_{b \bar{b}}^{S P}+X$ & $72 \pm 12_{\mathrm{sc}} \pm 5_{\mathrm{pdf}} \mathrm{mb}$ & $370 \pm 75_{\mathrm{sc}} \pm 30_{\mathrm{pdf}} \mathrm{mb}$ & $110 \pm 25_{\mathrm{sc}} \pm 5_{\mathrm{pdf}} \mathrm{mb}$ \\
$\sigma_{b \bar{b}, b \bar{b}, b \bar{b}+X}^{T P S}$ & $0.084 \pm 0.045_{\text {tot }} \mu \mathrm{b}$ & $11 \pm 7_{\text {tot }} \mu \mathrm{b}$ & $17 \pm 11_{\text {tot }} \mu \mathrm{b}$ \\
\hline
\end{tabular}

The TPS processes, although not observed so far, have visible cross sections for charm and bottom in pp and pA collisions at LHC and FCC energies. At the highest c.m. energies reached in collisions of cosmic rays with the nuclei in the upper atmosphere, the TPS cross section for triple charm-pair production equals the total pAir inelastic cross section, indicating that the average number of $c \bar{c}$-pairs produced in multiple partonic interactions is above unity.

\section{Conclusions}

We have derived simple formulas to compute the cross-sections for the simultaneous perturbative production of particles in double-, triple-, and in general n-parton scatterings, from the corresponding single-parton scattering cross-sections in p-p, p-A, and A-A collisions. Estimates have been presented for DPS and TPS production of heavy-quarks and quarkonia at LHC energies. The results presented here emphasize the importance of having a good understanding of multiparton interactions in hadronic collisions at current and future colliders, both as genuine probes of QCD phenomena and as backgrounds for searches of new physics in rare final-states with multiple heavyparticles, and their role in ultrarelativistic cosmic-ray interactions in the atmosphere.

Acknowledgments - The speaker would like to thank the Hard-Probes'18 organizers for the very generous welcome in Aix-Les-Bains. This work was supported by Russian Foundation for Basic Research (grant 18-02-00155).

\section{References}

[1] D. d'Enterria and A. M. Snigirev, arXiv:1708.07519 [hep-ph], Adv. Ser. Direct. High Energy Phys. 29 (2018) 159.

[2] D. d'Enterria and A. M. Snigirev, Phys. Rev. Lett. 118 (2017) 122001.

[3] D. d'Enterria and A. M. Snigirev, Eur. Phys. J. C 78 (2018) 359.

[4] M. Strikman and D. Treleani, Phys. Rev. Lett. 88 (2002) 031801.

[5] D. d'Enterria and A. M. Snigirev, Phys. Lett. B 718 (2013) 1395.

[6] D. d'Enterria and A. M. Snigirev, Phys. Lett. B 727 (2013) 157.

[7] D. d'Enterria and A. M. Snigirev, Nucl. Phys. A 931 (2014) 303.

[8] D. d'Enterria and A. M. Snigirev, Nucl. Phys. A 932 (2014) 296. 